\title{
Application of mathematical programming to prioritising interdependent Defence investment programs
}

\author{
$\underline{\text { Y.J. Wang }}{ }^{\text {a }}$ \\ a Joint Warfare Mathematical Science, Joint and Operations Analysis Division, Defence Science and \\ Technology Group, Canberra, Australian Capital Territory \\ Email: yue-jin.wang@,dst.defence.gov.au
}

\begin{abstract}
Defence capability planning often requires prioritising a list of candidate programs/projects/products to determine the portfolio of investments that provide the best value for money given resource constraints and uncertainty, organisational constraints, and program interdependency constraints. Many prioritising methodologies such as ranking models, scoring models and the analytic hierarchy process (AHP) may work well for programs that are predominantly independent of one another. When the programs are interdependent, those methods fail to explicitly capture dependencies between programs and the resource uncertainty.
\end{abstract}

This paper explores the use of a mathematical programming methodology for prioritising interdependent Defence programs. A service-based interrelationship identification method is used to measure the different levels of interdependencies between programs. Resource uncertainty is modelled through variations of possible budget limitations. Strategic objectives are linked by multiple scenarios with different weights. For different application situations, two mathematical models, the Quadratic Knapsack Model (QKM) and the Budget Scenario Model (BSM), are proposed to formulate the optimisation problem for prioritising interdependent programs. Algorithms and properties of the proposed optimisation models are further discussed through the application of the models to two simple Defence capability prioritisation problems. The preliminary results have shown the models' potential applications in support of Defence capability planning.

Keywords: $\quad$ Mathematical programming, interdependency, prioritisation 
Wang, Application of mathematical programming to prioritising interdependent Defence investment programs

\section{INTRODUCTION}

Defence's focus on a joint and integrated approach to the development of future Defence capability involves with the complexity of interdependencies among the investment programs/projects/products. One of the challenges in the Defence capability planning process is prioritising a list of candidate programs/projects to determine the portfolio of investments that provide the best value for money given resource constraints and uncertainty, organisational constraints, and program interdependency constraints. Many prioritising methodologies such as ranking models, scoring models and the analytic hierarchy process (AHP) may work well for programs that are predominantly independent of one another. When the programs are interdependent, those methods fail to explicitly capture the interdependencies between programs and the resource uncertainty (Dickson et al (2001); Order (2009)).

The complexity introduced by the program interdependencies requires a new integrated decision-support tool to assist in the process of prioritising Defence investment programs. The existing prioritising models either completely ignore the interdependencies or treat them as binary relationships, in which 0 or 1 values are assigned to the interdependent program/project pairs. Dickson et al (2001) used the binary representation of the project interdependencies in the nonlinear integer objective function with linear budget constraints while Greiner et al (2003) represented the binary interdependency relationships as additional linear constraints in their linear 0-1 integer programming model. Although the binary representation methods can effectively address the existence of the project interdependency, they have limitations in modelling the variable levels of interdependencies as the strength of interdependencies among programs may vary from large to insignificant.

This paper explores the use of a mathematical programming methodology for prioritising interdependent Defence programs. A service-based interrelationship identification method is used to measure the different levels of interdependencies between programs. Resource uncertainty is modelled through variations of possible budget limitations. Strategic objectives are linked by multiple scenarios with different weights. For different application situations, two mathematical models, the Quadratic Knapsack Model (QKM) and the Budget Scenario Model (BSM), are proposed to formulate the optimisation problem for prioritising interdependent programs. Algorithms and properties of the proposed optimisation models are further discussed through the application of the models to two simple Defence capability prioritisation problems. The preliminary results have shown the models' potential applications in support of Defence capability planning.

\section{MEASURING PROGRAM INTERDEPENDENCIES USING SCMILE FRAMEWORK}

In the current Defence acquisition process, a program may include many projects (future systems) and products (existing systems). Thus the interdependencies between programs are reflected by the projects/products interdependencies in a networked system of systems (SoS) manner. For simplicity, we use system to refer to a project or a product. In a joint and integrated operational environment, the relationships between systems are characterised as services, where one system is the provider of the service, and the other is the consumer. These services may be categorised as Sensing(S), Command and Control (C), Physical Mobility (M), Information Mobility (I), Logistics and Support (L), and Engagement (E), or simply SCMILE as described in Lowe (2015).

Under the SCMILE Framework, Subject Matter Experts (SMEs) are asked to identify and quantify the interrelationships between providers and consumers of the services. To represent the strength of the interdependencies, a five-point "Maturity Model" (Christensen (2012)) was used to measure the strength of the interdependencies between systems based on SME's assessment on the criticality of services of "mission success".

A brief description of each level of measuring the strength of interdependencies is given by Christensen (2012) as follows:

Level 0 - Independent: The systems do not have any influence over each other.

Level 1 -Tangential: Systems have a largely insignificant bearing on each other with respect to an Interdependency Factor. With some adjustment, the system could continue to function even if other tangentially connected systems were removed.

Level 2-Associated: The system is dependent or interdependent in ways that would result in the system being significantly affected if the associated system were disabled.

Level 3 -Dependent: The system is strongly connected to another system. The system would be severely impacted if a depended-upon system were removed or adversely affected.

Level 4 -Mandatory: The system requires a connected system in order to function. The program may share critical resources with another system or depend upon the other for basic operational capability. The system cannot survive if the depended-upon system is removed or severely affected. 
Wang, Application of mathematical programming to prioritising interdependent Defence investment programs

\section{INTEGRATING SCMIL INTERDEPENDENCY DATA INTO PROGRAM OPTIMISATION MODELLING}

We investigate a methodology to integrate the quantified SCMILE interdependency data into program/project prioritisation modelling. Assume that a multi-criteria decision analysis (MCDA) method has been applied to evaluate the "values" of each system, according to its roles aligned with strategic objectives.

Given $n$ interdependent systems, $\mathrm{P}_{1}, \ldots \mathrm{Pn}$, each has a capability value $v_{i}$, and a certain budget $c_{i}$, we aim to select a subset of systems which can achieve maximum capability values and satisfy the budget constraints B. The strength of interdependencies between systems is identified by means of the SCMILE Framework. For each service of SCMILE, the interdependency data can be represented as an $n \times n$ Dependency Matrix $D=\left(d_{i j}\right)$. Each element in $\mathrm{D}, d_{i j}$ varies from zero to four, representing the level of dependency that system $i$ has on system $j$. A value of zero implies that the system $i$ is entirely independent of system $\mathrm{j}$. A non-zero value of $d_{i j}$ implies that system $i$ has a certain level of dependency on system $j$.

Assume that a system $i$ can achieve its "independent" capability at $\alpha_{i}$ level (in percentage, $0 \leq \alpha_{i} \leq 1$ ) of its capability value $v_{i}$, the remaining part of the full capability, $\left(1-\alpha_{i}\right)$, should be attributable to its dependent systems. Based on the SCMILE data and its derived Dependency Matrix D, the percentage dependency component of system $j$ on system $i$ can be calculated as:

$$
\beta_{i j}=\left(1-\alpha_{i}\right)\left(\frac{d_{i j}}{\sum_{k=1}^{n} d_{i k}}\right)
$$

Then, we have two optimisation models for prioritising systems as follows:

\section{(1) Quadratic Knapsack Model (QKM)}

When only a generic strategic scenario is considered and one set of system values are available, then the prioritisation problem can be formulated as a variant of Quadratic Knapsack Model (Kellerer et al (2004); Taylor (2015)) as follows:

Maximise $\quad \sum_{i=1}^{n} v_{i}\left(\alpha_{i}+\sum_{j=1}^{n} \beta_{i j} x_{j}\right) x_{i}$

Subject to $\quad \sum_{i=1}^{n} c_{i} x_{i} \leq B$

$$
x_{i} \in\{0,1\}
$$

where $x_{i} \in\{0,1\}$ is the integer decision variable, $x_{i}=1$ means the system $i$ is selected, otherwise system $i$ is not selected. In formulating QKM, we have assumed that all systems are synergistic, that is, the value of individual systems can be additive and enhanced when they work together.

When there is no interdependency between systems, $d_{i j}=0, \alpha_{i}=1, \beta_{i j}=0(i=1, . . \mathrm{n} ; j=1, . . \mathrm{n})$, the objective function (2) becomes a linear function and the model becomes a linear Knapsack problem (Greiner et al (2003)).

For QKM, the SCMILE data can be integrated into the objective function (1) either as a maximum matrix over all services (i.e. the worst scenario in terms of interdependencies) or as a single dependency matrix individually.

\section{(2) Budget Scenario Model (BSM)}

When multiple strategic scenarios are considered and the system values for each scenario are given, then the prioritisation problem can be formulated as a variant of Budget Scenario Model (Taylor (2015)) as follows:

Maximise $\quad \sum_{i=1}^{m} s_{i} \max _{1 \leq j \leq n}\left(v_{i j}\left(\alpha_{j}+\sum_{k=1}^{n} \beta_{j k} x_{k}\right) x_{j}\right)$ 
Wang, Application of mathematical programming to prioritising interdependent Defence investment programs

Subject to

$$
\begin{aligned}
& \sum_{i=1}^{n} c_{i} x_{i} \leq B \\
& x_{i} \in\{0,1\}
\end{aligned}
$$

where $m$ is the number of scenarios, each scenario has a probability of occurring $s_{i},(i=1, \ldots m)$, and system $j$ in scenario $i$ has a value $v_{i j}$.

To integrate SCMILE interdependency data into the BSM objective function (5), a single service may be regarded as a "scenario". It is noticed that the BSM objective function (5) is a non-linear non-smooth function. Although the BSM problem is NP-hard, it can be solved with a polynomial time approximation algorithm (Taylor (2015)).

\section{APPLICATION EXAMPLES}

\subsection{Example of Quadratic Knapsack Model (QKM)}

To test applications of the proposed QKM model, we use a small example of a Defence systems selection problem as described in Greiner et al (2003). Fifteen systems are considered and the total fund available is $\$ 196.46 \mathrm{M}$. The "value" of each system was evaluated by means of the Analytical Hierarchy Process and listed in the following Table 1.

In Table 1, the selected set of systems is called a portfolio. For the available fund of $\$ 196.46 \mathrm{~m}$, we first assumed all systems are independent, that is, $\mathrm{D} \__{-}=\left(d_{i j}\right), d_{i j}=0$ (for all $i, j=1,2, \ldots 15$ ). Then we use the interdependency matrices D_S, D_C, D_M, and D_E to test the effect of the system interdependencies. The details of those SCMILE matrices are included in the Appendix. D_Max is a matrix taking the maximum values of D_S, D_C, D_M, D_E as its elements. Table 1 presents a comparison of the optimal system selection results for different interdependency matrices. $x_{i}=1$ indicates the system $i$ was selected, while a " 0 'indicates the system $i$ was not selected.

\begin{tabular}{|c|c|c|c|c|c|c|c|c|c|c|c|c|c|c|}
\hline \multirow[b]{2}{*}{ Project } & \multirow{2}{*}{$\begin{array}{l}\text { Cost } \\
\text { \$m }\end{array}$} & \multirow[b]{2}{*}{ Value } & \multicolumn{2}{|c|}{ D_0 } & \multicolumn{2}{|r|}{ D_S } & \multicolumn{2}{|c|}{ D_C } & \multicolumn{2}{|c|}{ D_M } & \multicolumn{2}{|c|}{ D_E } & \multicolumn{2}{|c|}{ D_Max } \\
\hline & & & $x_{i}$ & Value & $x_{i}$ & Value & $x_{i}$ & Value & $x_{i}$ & Value & $x_{i}$ & Value & $x_{i}$ & Value \\
\hline P1 & 34.5 & 0.073 & 1 & 0.073 & 1 & 0.073 & 1 & 0.062 & 1 & 0.073 & 0 & 0.000 & 0 & 0.000 \\
\hline P2 & 3 & 0.069 & 1 & 0.069 & 1 & 0.054 & 1 & 0.069 & 1 & 0.069 & 1 & 0.069 & 1 & 0.038 \\
\hline P3 & 16.3 & 0.07 & 1 & 0.070 & 0 & 0.000 & 1 & 0.070 & 1 & 0.070 & 0 & 0.000 & 0 & 0.000 \\
\hline P4 & 32.8 & 0.092 & 1 & 0.092 & 1 & 0.092 & 1 & 0.085 & 1 & 0.092 & 1 & 0.092 & 1 & 0.085 \\
\hline P5 & 44.3 & 0.07 & 0 & 0.000 & 0 & 0.000 & 0 & 0.000 & 0 & 0.000 & 1 & 0.070 & 1 & 0.061 \\
\hline P6 & 13.86 & 0.066 & 1 & 0.066 & 1 & 0.066 & 1 & 0.058 & 1 & 0.066 & 1 & 0.059 & 1 & 0.059 \\
\hline P7 & 4.71 & 0.056 & 1 & 0.056 & 1 & 0.056 & 1 & 0.051 & 0 & 0.000 & 1 & 0.051 & 1 & 0.051 \\
\hline P8 & 4.65 & 0.055 & 1 & 0.055 & 1 & 0.055 & 1 & 0.050 & 1 & 0.055 & 1 & 0.055 & 1 & 0.044 \\
\hline P9 & 10.5 & 0.052 & 0 & 0.000 & 1 & 0.052 & 0 & 0.000 & 1 & 0.052 & 1 & 0.052 & 1 & 0.052 \\
\hline P10 & 3.3 & 0.068 & 1 & 0.068 & 1 & 0.068 & 1 & 0.064 & 1 & 0.068 & 1 & 0.063 & 1 & 0.062 \\
\hline P11 & 10.2 & 0.062 & 1 & 0.062 & 1 & 0.062 & 1 & 0.052 & 1 & 0.062 & 1 & 0.062 & 1 & 0.062 \\
\hline P12 & 30.6 & 0.072 & 1 & 0.072 & 1 & 0.072 & 1 & 0.072 & 1 & 0.072 & 1 & 0.072 & 1 & 0.072 \\
\hline P13 & 15.1 & 0.069 & 1 & 0.069 & 1 & 0.069 & 1 & 0.069 & 1 & 0.069 & 1 & 0.062 & 1 & 0.062 \\
\hline P14 & 16.46 & 0.065 & 1 & 0.065 & 1 & 0.065 & 1 & 0.059 & 1 & 0.065 & 1 & 0.058 & 1 & 0.060 \\
\hline P15 & 4.8 & 0.061 & 1 & 0.061 & 1 & 0.061 & 1 & 0.061 & 1 & 0.061 & 1 & 0.053 & 1 & 0.053 \\
\hline \multicolumn{3}{|c|}{ Portfolio Value } & & 0.878 & & 0.845 & & 0.822 & & 0.874 & & 0.818 & & 0.761 \\
\hline \multicolumn{3}{|c|}{ Requested Fund \$m } & & 190.28 & & 184.48 & & 190.28 & & 196.07 & & 194.28 & & 194.28 \\
\hline
\end{tabular}

Table 1. Comparison of portfolio optimisation results

The optimisation model was computed in Microsoft Excel Solver (non-linear solver is used). The computational results in Table 1 show that 
Wang, Application of mathematical programming to prioritising interdependent Defence investment programs

(1) If all systems are independent (D_0), the proposed Quadratic Knapsack Model becomes a linear Knapsack problem and the optimisation results are exactly the same as those obtained by Greiner et al (2003).

(2) When the interdependency data are integrated with the QKM, the optimisation results are affected by the interdependencies. The interdependent systems appear to stay together either in or out of the portfolio. For example, for interdependency matrix D_E, there are seven systems with strong dependencies on P5 and those systems form a group to stay in the portfolio, even though P5 has the largest cost.

(3) It is noticed that for all five different interdependency cases, at least 11 of the $15(73 \%)$ system alternatives are identical with those obtained by the optimisation model without interdependency data. Among them, D_C has the same results with D_0. Although some portfolio selections are the same, the QKM model presents different values for the selected systems. For example, D_E selected P6 with a value of 0.59, but P6's original value is 0.66 . The partial value of the systems selected by QKM are due to the effects of its dependent systems as the system's full value can be achieved only if all of its dependent systems are selected. The QKM results provide the decision maker with a quantitative measure of the effects of interdependencies and with additional insight regarding the robustness of recommended portfolio options

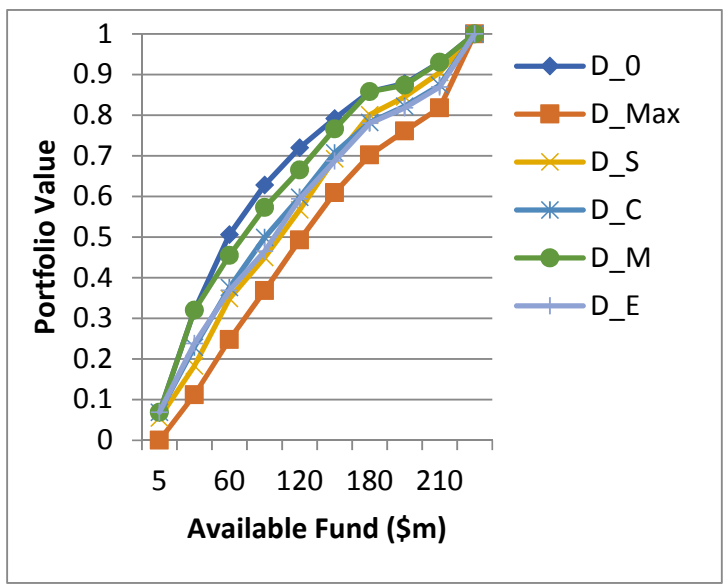

In the above Table 1 , it is assumed that the maximum budget available is known to be $\$ 196.46 \mathrm{~m}$. Figure 1 presents the changes of the portfolio values against different available funds using QKM with different SCMILE interdependency matrices. Because the QKM model incorporates the aggregated effects of interdependencies, the change of the portfolio value appears to be reasonably smooth. A comparison with individual interdependency matrices shows that the QKM model with maximum interdependency matrix D_Max produces the lowest portfolio values while "zero" interdependency matrix D_0 provide the highest portfolio values.

Figure 1. Portfolio value versus budget- QKM

\subsection{Example of Budget Scenario Model (BSM)}

The Budget Scenario Model (BSM) is a budget -value problem in which the best value for a range of budgets is sought (Taylor (2015)). A list of initiatives (or systems) is provided each with an anticipated cost. Each initiative/system is given a value (score) against a number of scenarios with an occurring probability. BSM aims to find a subset of initiatives whose total cost is bounded by an available fund to achieve the maximum value for all possible scenarios. In BSM modelling, it is assumed that only one of the scenarios will occur (with a given probability) and only the best initiative chosen for that scenario will be applied at the time the scenario occurs (Order (2009)). There is no synergy requirement among the initiatives/systems.

We use an example of prioritising military initiatives described in Order (2009). The problem is composed of fifteen initiatives in four scenarios. The details of data are listed in Table 2. In Order (2009), it is assumed a few binary interdependencies such as "Initiative $O$ requires initiative $K$ in Scenario I" and "Initiatives J,L, and $H$ all require Initiative B in Scenario 3". By means of the SCMILE data structure and the BSM model in (5)-(7), those binary interdependencies (D_Bin) among the initiatives can be easily represented by dependency matrices. For example, "Initiative O requires initiative K in Scenario 1" means that for the dependency matrix in which Scenario 1 is represented, element $d_{1,14}=4$ as $\mathrm{O}$ is in the $1^{\text {st }}$ row and $\mathrm{K}$ is in the $14^{\text {th }}$ column. Here we have assumed that each dependency matrix represents one scenario. For test purposes, we also applied BSM modelling for the situations of independent initiatives (D_0) and non-binary interdependency D_SCME, in which the interdependency for each scenario is represented by D_S, D_C, D_M, and D_E individually.

Computationally, optimisation modelling was implemented in the Microsoft Excel Solver. An evolutionary solver is used for solving the BSM problem as the modelling contains a non-smooth objective function (5). 
Table 2. Budget Scenario Model - Data sheet from Order (2009)

\begin{tabular}{|c|c|c|c|c|c|}
\hline \multirow[b]{2}{*}{ Initiative } & \multirow[b]{2}{*}{ Cost } & \multicolumn{4}{|c|}{ Value $\left(\mathrm{S}_{\mathrm{i}}=\right.$ Scenario $\left.i\right)$} \\
\hline & & $\begin{array}{c}S_{1} \\
(30 \%)\end{array}$ & $\begin{array}{c}\mathrm{S}_{2} \\
(10 \%)\end{array}$ & $\begin{array}{c}S_{3} \\
(40 \%)\end{array}$ & $\begin{array}{c}\mathrm{S}_{4} \\
(20 \%)\end{array}$ \\
\hline 0 & 1 & 10 & 1 & 8 & 2 \\
\hline M & 1 & 7 & 1 & 3 & 4 \\
\hline N & 3 & 6 & 3 & 5 & 6 \\
\hline D & 4 & 8 & 7 & 1 & 2 \\
\hline J & 7 & 4 & 3 & 7 & 9 \\
\hline I & 6 & 8 & 7 & 4 & 1 \\
\hline B & 8 & 8 & 8 & 3 & 10 \\
\hline L & 11 & 10 & 5 & 6 & 9 \\
\hline $\mathrm{E}$ & 13 & 8 & 4 & 9 & 7 \\
\hline A & 12 & 7 & 2 & 9 & 1 \\
\hline $\mathrm{H}$ & 14 & 1 & 5 & 9 & 9 \\
\hline G & 17 & 9 & 5 & 8 & 4 \\
\hline c & 14 & 5 & 7 & 5 & 7 \\
\hline K & 20 & 6 & 4 & 9 & 4 \\
\hline $\mathrm{F}$ & 16 & 1 & 7 & 9 & 1 \\
\hline
\end{tabular}

Table 3. Comparison of optimisation results Budget Scenario Model

\begin{tabular}{|c|c|c|c|c|c|c|}
\hline \multirow[b]{2}{*}{ Budget } & \multicolumn{2}{|c|}{ D_SCME } & \multicolumn{2}{|c|}{ D_0 } & \multicolumn{2}{|c|}{ D_Bin } \\
\hline & Value & Selection & Value & Selection & Value & Selection \\
\hline 1 & 6.7 & 0 & 6.7 & 0 & 4.2 & $M$ \\
\hline 2 & 7.1 & $\mathrm{M}, \mathrm{O}$ & 7.1 & $\mathrm{M}, \mathrm{O}$ & 6.2 & $\mathrm{M}, \mathrm{O}$ \\
\hline 4 & 7.6 & $\mathrm{~N}, \mathrm{O}$ & 7.7 & $\mathrm{~N}, \mathrm{O}$ & 6.5 & $\mathrm{~N}, \mathrm{O}$ \\
\hline 5 & 7.6 & & 7.7 & & 6.8 & $\mathrm{M}, \mathrm{N}, \mathrm{O}$ \\
\hline 6 & 7.6 & & 7.7 & & 7.1 & $D, M, O$ \\
\hline 8 & 8 & $\mathrm{~J}, \mathrm{O}$ & 8.3 & $\mathrm{~J}, \mathrm{O}$ & 7.5 & $\mathrm{D}, \mathrm{N}, \mathrm{O}$ \\
\hline 9 & 8.1 & $\mathrm{~J}, \mathrm{M}, \mathrm{O}$ & 9 & $\mathrm{~B}, \mathrm{O}$ & 8.4 & $\mathrm{~B}, \mathrm{O}$ \\
\hline 10 & 8.1 & & 9 & & 8.4 & \\
\hline 11 & 8.2 & $\mathrm{~J}, \mathrm{~N}, \mathrm{O}$ & 9 & & 8.4 & \\
\hline 12 & 8.4 & $D, J, O$ & 9 & & 8.5 & $\mathrm{~L}, \mathrm{O}$ \\
\hline 16 & 8.5 & $\mathrm{H}, \mathrm{M}, \mathrm{O}$ & 9 & & 8.7 & $D, L, O$ \\
\hline 18 & 8.6 & $\mathrm{H}, \mathrm{N}, \mathrm{O}$ & 9 & & 8.7 & \\
\hline 19 & 8.8 & & 9.1 & $\mathrm{D}, \mathrm{H}, \mathrm{O}$ & 8.7 & \\
\hline 20 & 8.8 & & 9.1 & $\mathrm{D}, \mathrm{H}, \mathrm{O}$ & 9.0 & \\
\hline 21 & 8.8 & & 9.4 & $A, L, O$ & 9.0 & \\
\hline 23 & 8.9 & $\mathrm{~B}, \mathrm{H}, \mathrm{O}$ & 9.4 & & 9.0 & \\
\hline 24 & 9.1 & $\mathrm{~J}, \mathrm{~F}, \mathrm{O}$ & 9.4 & & 9.0 & \\
\hline 27 & 9.1 & & 9.4 & & 9.1 & $A, D, L$ \\
\hline 29 & 9.1 & & 9.4 & & 9.4 & $\mathrm{~B}, \mathrm{~K}, \mathrm{O}$ \\
\hline 30 & 9.1 & & 9.4 & & 9.4 & \\
\hline 31 & 9.1 & & 9.4 & & 9.4 & \\
\hline
\end{tabular}

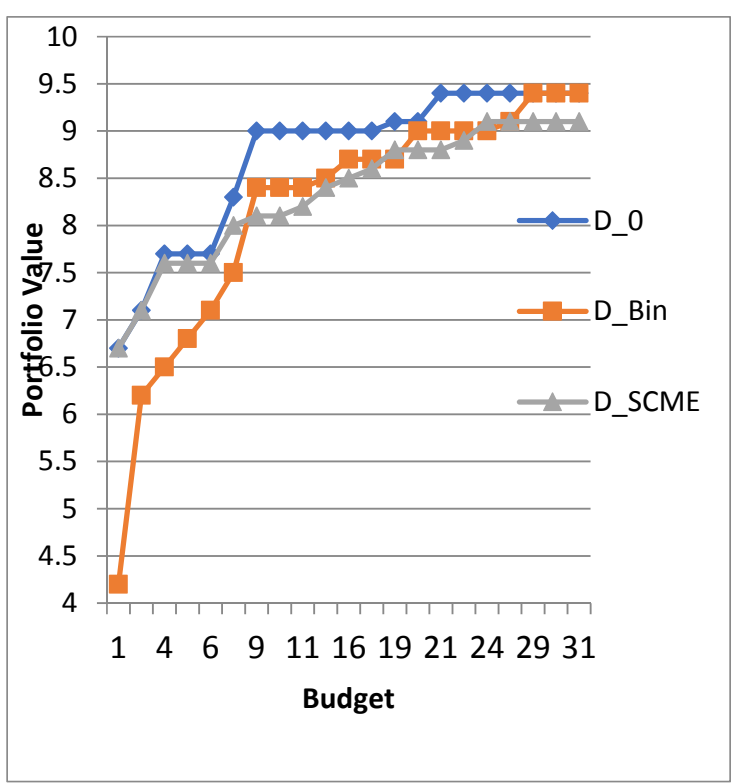

Figure 2. Portfolio value achieved versus increased budget - BSM.

The BSM results presented in Figure 2 and Table 3 have shown that

(1) The interdependency data have significant effects on the BSM initiatives optimisation. For the same budget constraint, even though the selections of initiatives are the same, the portfolio values may be different because of different interdependencies in individual scenarios. For example, when budget $\mathrm{B}=4, \mathrm{D} \_0, \mathrm{D} \_$Bin, and D_SCME all have the same selection of $\mathrm{N}$ and $\mathrm{O}$, but the maximum values used in the objective function (5) are different: for scenario 1, D_Bin can only takes initiative N's value of 6 as the maximum value because $\mathrm{O}$ depends on $\mathrm{K}$, while D_0 and D_SCME can take O's value of 10 as the maximum value.

(2) Like the QKM, the grouping effects of the interdependencies also appear in BSM modelling. It chooses groups of initiatives that are either interdependent or independent. For example, for

D_SCME, initiative $\mathrm{J}$ and $\mathrm{O}$ are chosen together a few times because there are independent of each other in D_S,D_C,D_E, and are highly interdependent in D_M.

(3) As the BSM modelling has a non-smooth objective function, the effects of interdependences are not aggregated. Table 3 shows that the selection of initiatives changes significantly even for a small amount of variations in budget. Figure 2 displays the "stair-type" change of the portfolio value versus the change of budget.

\section{CONCLUSION AND FURTHER WORK}

This study proposes two mathematical programming models, Quadratic Knapsack Model (QKM) and Budget Scenario Model (BSM), to integrate the effects of interdependency data into the program prioritisation problem. The algorithms and mathematical properties of the above models are further analysed and discussed with two numerical examples. These illustrative examples have demonstrated the feasibility of the proposed mathematical models in producing optimisation solutions in prioritising interdependent systems. 
With the primary aim of integrating the available SCMILE interdependency data into the Defence prioritisation problem, this study may form a basis for the further development of an extensive value-based system modelling. Further work may include

(1) Application of the proposed models to a Defence capability development problem, including client engagement, data collection, and model refinement;

(2) Extending the proposed models to Defence system effectiveness modelling, including dealing with system uncertainty and the development of decision-support tool set.

\section{ACKNOWLEDGEMENTS}

The author would like to thank Dr Richard Taylor, Dr Jerzy Jagiello, and Dr Minh-Tuan Nguyen for their helpful comments that improved the article.

\section{REFERENCES}

Christensen M.B. (2011). A Method for Measuring Programmatic Dependency and Interdependency between DOD Acquisition Programs, Thesis, AFIT/GSE/ENV/11-D02DL, Air Force Institute of Technology, WrightPatterson Air Force Base, Ohio.

Dickinson M, Thornton A.C., and Graves S. (2001). Technology Portfolio Management: Optimizing Interdependent Projects Over Multiple Time Periods, IEEE Transaction on Engineering Management, 2001, 48(4), 518-527.

Greiner M A, Fowler J.W, Shunk D. L, Carlyle W. M., and McNutt R. (2003). A Hybrid Approach Using the Analytic Hierachy Process and Integer Programming to Screen Weapon Systems Projects, IEEE Transaction on Engineering Management, 50(2), 192-203.

Kellerer H., Pferschy U., and Pisinger D. (2004). Knapsack Problems, Springer, Berlin.

Lowe D. (2015), A Quick Overview of SCMILE Services and Their Evolution, DSTO internal report.

Order N.( 2009). The Priority List-Revised, PHALANX- The Bulletin of Military Operations Research, 42(4), 2334.

Taylor R. (2015). Algorithmic Complexity of Two Defence Budget Problems, The $21^{\text {st }}$ International Congress on Modelling and Simulation (MODSIM2015), Gold Coast, Australia, Dec 1-4.

\section{APPENDIX: SCMLE DATA USED IN THIS STUDY}

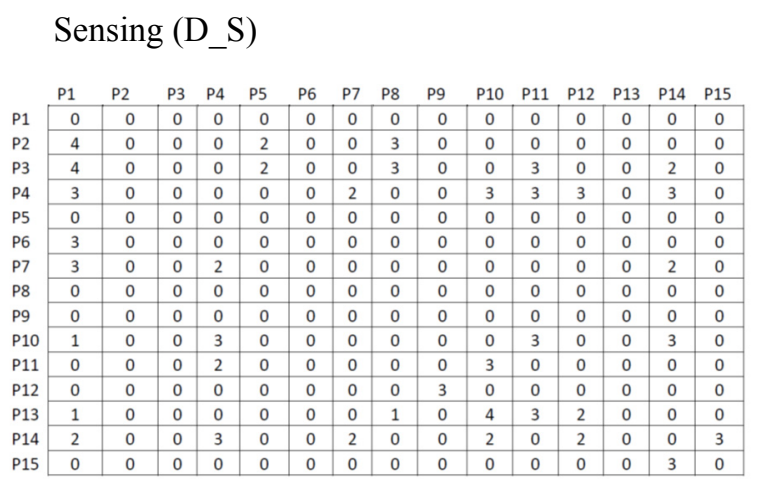

Physical Mobility (D_M)

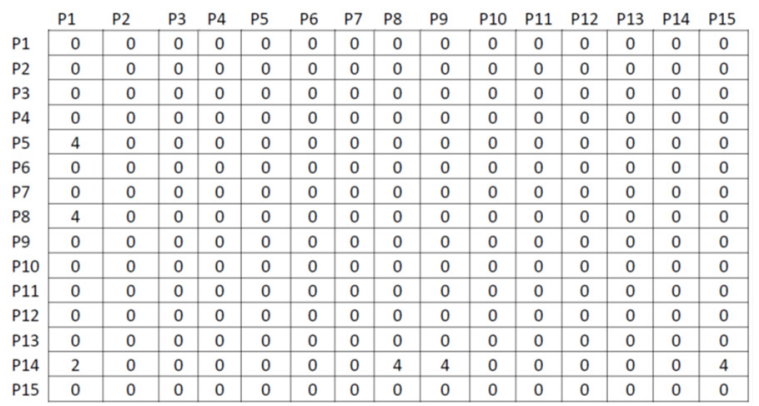

Command \&Control (D_C)

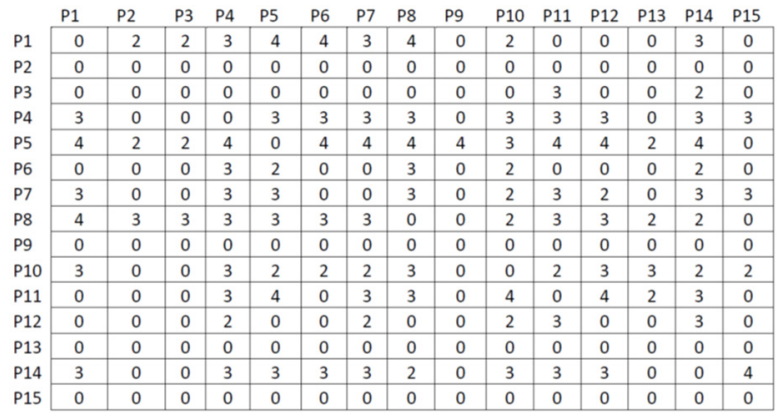

Engagement (D_E)

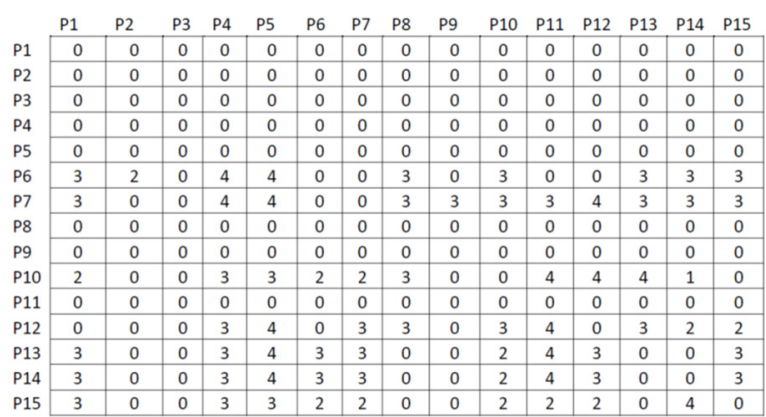

